\title{
Detached-Eddy Simulation of Trailing-Edge (TE) Cutback Turbine Blade Cooling
}

\author{
Marwan Effendy ${ }^{1}$ Yufeng $\mathrm{Yao}^{2}$, and Denis R. Marchant ${ }^{3}$ \\ ${ }^{1}$ Department of Mechanical Engineering, Universitas Muhammadiyah Surakarta, Jl. A.Yani \\ Surakarta, Indonesia, 57102 \\ ${ }^{2}$ Department of Engineering Design and Mathematics, University of the West of England, \\ Bristol BS16 1QY, UK \\ ${ }^{3}$ School of Aerospace \& Aircraft Engineering, Kingston University, Friars Avenue, London \\ SW15 3DW, UK
}

\begin{abstract}
This research evaluates the cooling performance of trailing-edge cutback for gas turbine blade. By using DES based on SST k- $\omega$ turbulence model, numerical investigations were performed at two steps: first, to validate simulation results from an existing TE cutback cooling with staggered pin-fin arrays inside the cooling passage against experimental measurement. Three types structured mesh from coarse $\left(\Delta \mathrm{y}^{+}=0.74\right)$ to fine $\left(\Delta \mathrm{y}^{+}=\right.$ 1.22) were evaluated during this step; second, to investigate the TE cutback cooling performance on various blowing ratios. Simulations were performed by keeping the same initials and boundary conditions as the experiment. The result indicates that validation can be considered acceptable by controlling grid quality resolution near wall regions. Both computational data of the adiabatic film-cooling effectiveness and the discharge coefficient are in good agreement with available experimental measurements. The averaged film-cooling effectiveness along the cutback region is highly influenced by the blowing ratios, which is to be related to the turbulent flow structures formed at the mixing region as the impact of coolant flow ejection. The increase of coolant jet velocity triggers the heat transfer process up to the downstream region of TE cutback cooling.
\end{abstract}

\section{Introduction}

Gas turbine engines require a very high inlet temperature in order to maximise the power output to achieve a high thermal efficiency. Modern gas turbines typically operate at temperatures of around $1,200-1,500^{\circ} \mathrm{C}$ [1]. The temperature can be up

${ }^{1}$ Corresponding author: Marwan.Effendy@ums.ac.id 
to $2,000^{\circ} \mathrm{C}$ for a double engine power in aircraft gas turbines [2]. It is significantly beyond the melting temperatures for turbine vane and blade materials. High turbine inlet temperature causes structural strength degradation that eventually results in vane and blade break down. It causes a serious implication on durability and safety. Therefore, turbine blades/vanes must be cooled down to the levels that are significantly below the melting point of the material using internal and external cooling techniques. The cooling can be done by combining convection and film cooling. A comprehensive review paper by Han et al. [2, 3] summarised recent advancements in this field including both internal-cooling passage and external film cooling jets.

The trailing edge is the most critical part in a vane/blade component due to its geometrical constraint, in combination with aerodynamic, thermal and structural requirements. The trailing edge is particularly vulnerable to high turbine inlet temperatures because of its thin structure, which is susceptible to heavy mechanical and thermal stress. An effective blade TE cooling system should keep vane/blade temperature within its limit without affecting both mechanical strength and aerodynamic losses. Trailing-edge blades become one of the most interesting topics due to the conflict of interest between the need of cooling and aerodynamic requirements. Moreover, the heat-transfer enhancement around the downstream of trailing-edge and thermal-shield by film-cooling behind the lip creates more complicated issues.

Numerical investigations of TE cutback cooling can be found in open literature, but only little information explains the dynamic flow interaction between the internal cooling and the external cooling in a comprehensive manner. So far, most of the numerical studies employ the steady RANS model that does not capture well in terms of the turbulent flow mixing. Therefore, the predicted CFD data does not match with the measurement data. For instance, the use of steady RANS predicts ideal results, which ignores a decay of effectiveness as reported by Holloway et al. [4], Martini et al. [5], Effendy et al. [6], and Egorov et al. [7].

It is known that the use of unsteady RANS calculation is capable on capturing the turbulent flow structures at the mixing region as discovered by Medic et al. [8] and Joo et al. [9]. Unfortunately, it does not optimally simulate the flow interactions between the mainstream flow and the coolant. Consequently, CFD prediction of film-cooling effectiveness does not match with the experimental data as found by Holloway et al. [4], Egorov et al. [7], Medic et al. [8], and Joo et al. [9].

Other unsteady methods have been applied by eddy simulation in order to gain a better prediction on capturing turbulent flow structures of mixing. For example, Scale-Adaptive Simulation (SST-SAS) was used by Egorov et al. [7], DetachedEddy Simulation based on Spalart-Allmaras turbulence model was applied by Martini et al. [10] and Krueckel et al. [11], and Large-Eddy Simulation was utilized by Viswanathan et al. [12]] and Schneider et al. [13][14]. Moreover, Viswanathan et al. [15] employed eddy simulation for modelling forbody [16] and internal cooling [17]. A pressure-side bleed on the trailing edge with realistic turbine condition was numerically studied by Holloway et al. [18] for both steady and unsteady RANS.

Similar to TE cutback as experimentally investigated by Holloway et al. [4, 18] and Chen et al. [19], further numerical study has been undertaken by Medic et al. [8] using ANSYS-CFX commercial code. They applied the unsteady RANS with the shear-stress transport (SST) turbulence model computation using 1.25 million 
cells. In continuation of works from Medic et al. [8], Joo et al. [9] undertook their work using the Stanford University SUMB CFD code. They applied both the SSTRANS modelling and the SST-SAS (scale-adaptive simulation) for eddy-simulation on their numerical study. Based on their studies, both Medic et al. [8] and Joo et al. [9] noted that prediction by eddy simulation is appropriate with the available data of experiments, whereas steady/unsteady RANS predicts ideally the level of filmcooling effectiveness nearly unity along the trailing-edge surfaces, mainly for higher blowing ratios. The film-cooling effectiveness predicted by un-steady RANS was not consistent with tested data due to coolant air near the trailing-edge surface was protected from mixing process with main hot gas. A small chunk of main hot gas did not hit the bottom wall along the trailing-edge cutback. The over-prediction of film-cooling effectiveness by unsteady RANS was due to the deficiency of unsteady coherent energy at near wall of the TE cutback surfaces. Along this surface, both the total turbulence kinetic energy and the mean temperature predicted by unsteady RANS was significantly less than the eddy simulation. This finding indicated that there was a strong correlation between the level of turbulence kinetic energy and the profiles of the mean temperature, which caused the degree of mixing process. This research also noted that the three-dimensional (3D) vortex-shedding caused by the upper lip is a dominant aspect that influences a mixing flow over the TE cooling surface. Unsteadiness at the TE breakout was composed by two components namely coherent and broadband components. The coherent component was due to unsteadiness and the broadband component was denoted by a closure model [9].

In parallel with their experiments, Martini et al. $[5,20]$ evaluated numerically the performance of gas turbine blade trailing edge cutback cooling. Through studies of different TE configurations including "two-rows of long ribs" and "circular pinfin arrays" inside the cooling passage, Martini et al. [5] developed a multi-block structured mesh up to 0.89 million cells with average of $\Delta y^{+}<2$, and simulated on three different blowing ratio (i.e. $M=0.5,0.8$, and 1.1 ). This prediction found that a highly complex turbulent mixing process was not captured correctly using 1 model with CFX Tasc-Flow. There was a significant deviation of film-cooling effectiveness between CFD data and measurements, particularly in the middle of the trailing-edge cutback region. Further numerical study showed that the prediction could be improved by applying the Spalart-Allmaras turbulence model for DES simulation. This approach gave a detailed insight into the unsteady film mixing on the blade TE breakout region [10]. The results revealed that the adiabatic filmcooling effectiveness and the heat-transfer coefficient were in a good agreement between numerical prediction and experiments at all blowing ratios.

Schneider et al. [13, 14] investigated computationally the interrelationship between turbulent heat-flux and large coherent structures in a film-cooled TE cutback of gas turbine blades. Their large-eddy simulation was found that the blowing ratio and the flow regime of the cool gas flow influence both type and the strength of large-scale structures. The change in large coherent structures had a significant consequence to the dynamic mixing process at the breakout region. Dominant clockwise-rotating flow structures existed in the region of blowing ratio in which the counter-intuitive behaviour occurred. A large coherent structure increased the upstream-and-wall-directed turbulent heat-flux. This turbulent heatflux caused the enhanced thermal mixing process in the near wall region. 
Recently, Effendy et al. [21], demonstrated the capability of DES on the prediction of the cooling performance of a trailing edge cutback turbine blade cooling with various lip-thicknesses to slot height ratios $(t / H)$ referring to the work of Martini et al. [10, 20]. The DES study was based on the shear-stress transport (SST) $k-\omega$ turbulence model in order to simulate the dynamic flow interaction between internal coolant and mainstream flow. The discharge coefficient, the adiabatic film-cooling effectiveness and the vortex shedding frequency were in a good agreement between numerical prediction and measurements. With respect to the previous works, the present study focuses on the numerical study of blade trailing-edge cutback cooling, which is particularly important and challenging for the susceptibility of the blade damage due to higher local thermal loadings and a relatively thinner structure. The study concentrates on the interaction mixing between the internal cooling and the TE cutback cooling as the effect of blowing ratio $(\mathrm{M})$.

\section{Numerical Treatment}

\subsection{Physical problem and flow conditions.}

Numerical study considers an experimental configuration investigated by Martini et al. $[10,20]$. The computational domain includes main hot gas domain of stream wise, as published by Effendy et al. [21]. The coolant passage contains five rows of staggered-array cylindrical pins located at the coolant flow region. The arrays are fitted in a $10^{\circ}$ wedge-shaped duct, representing the shape of trailing edge that follow the angle of the coolant ejection slot. Spanwise pitch $(S / D)$ and streamwise pitch $\left(S_{x} / D\right)$ are kept the same ratio as the experiment. The blade trailing-edge model has a lip-thickness $(t)$ of $4.8 \mathrm{~mm}$, same as the coolant passage height $(H)$, thus the ratio of lip thickness to coolant passage height $(t / H)$ is 1 for this computational domain. The same $t / H$ ratio of a trailing-edge model has been tested and simulated in previous studies using eddy simulation based on Spalart-Allmaras model [10].

Initial flow and boundary conditions use the same values as applied for the Martini's experiment as seen in table 1.

Table 1. The experimental test conditions [20] .

\begin{tabular}{rlrl}
\hline & Mainstream flow (hot gas) & & Coolant flow (cold gas) \\
\hline$R e_{\mathrm{hg}}$ & $=250,000$ & $T_{\mathrm{c}^{\prime}}, L_{2}$ exit & $\begin{array}{l}=295-330 \mathrm{~K} \text { (cold gas } \\
\text { temperature at the exit slot) }\end{array}$ \\
$M a_{\mathrm{hg}}$ & $=0.125$ & & \\
$u_{\mathrm{hg}}$ & $=56 \mathrm{~m} / \mathrm{s}$ (inlet velocity) & & \\
$T u_{\mathrm{hg}}$ & $=7 \%$ & $T_{\mathrm{c}}, L_{1 \text { inlet }}$ & $=293 \quad \mathrm{~K} \quad($ cold gas \\
$T_{\mathrm{hg}}$ & $=500 \quad \mathrm{~K} \quad($ hot gas & $T u_{\mathrm{c}}$ & temperature \\
$P_{\mathrm{hg}}$ & temperature) & $M$ & $=5 \%$ \\
& $=105 \mathrm{kPa}$ (pressure) & & $=0.2-1.25$ (blowing ratio) \\
\hline
\end{tabular}




\subsection{Mesh generation}

The mesh for this computation was constructed by high quality grids with $\Delta \mathrm{y}^{+}<1$ on all surfaces, as shown in Fig. 1. In addition, the interesting area (i.e. adiabatic/protected wall surface) is constructed in higher quality meshes with average $\Delta \mathrm{y}^{+}<0.5$, as addressed by Nishino, et al. [22] in an effort to assure a sufficiently fine spatial resolution from unsteady phenomenon at the mixing region. As suggested by Spalart et al. [23][24] and Joo et al. [9], grid must be refined in each of the $x, y$ and $z$ directions in order to satisfy the blending factor as addressed by Menter et al. [25]. It is to be related the consistency of local grid spacing in the 3-D domain. The growth of grid spacing must be controlled linking to the requirement of blending factor $(F)$ for the DES method.

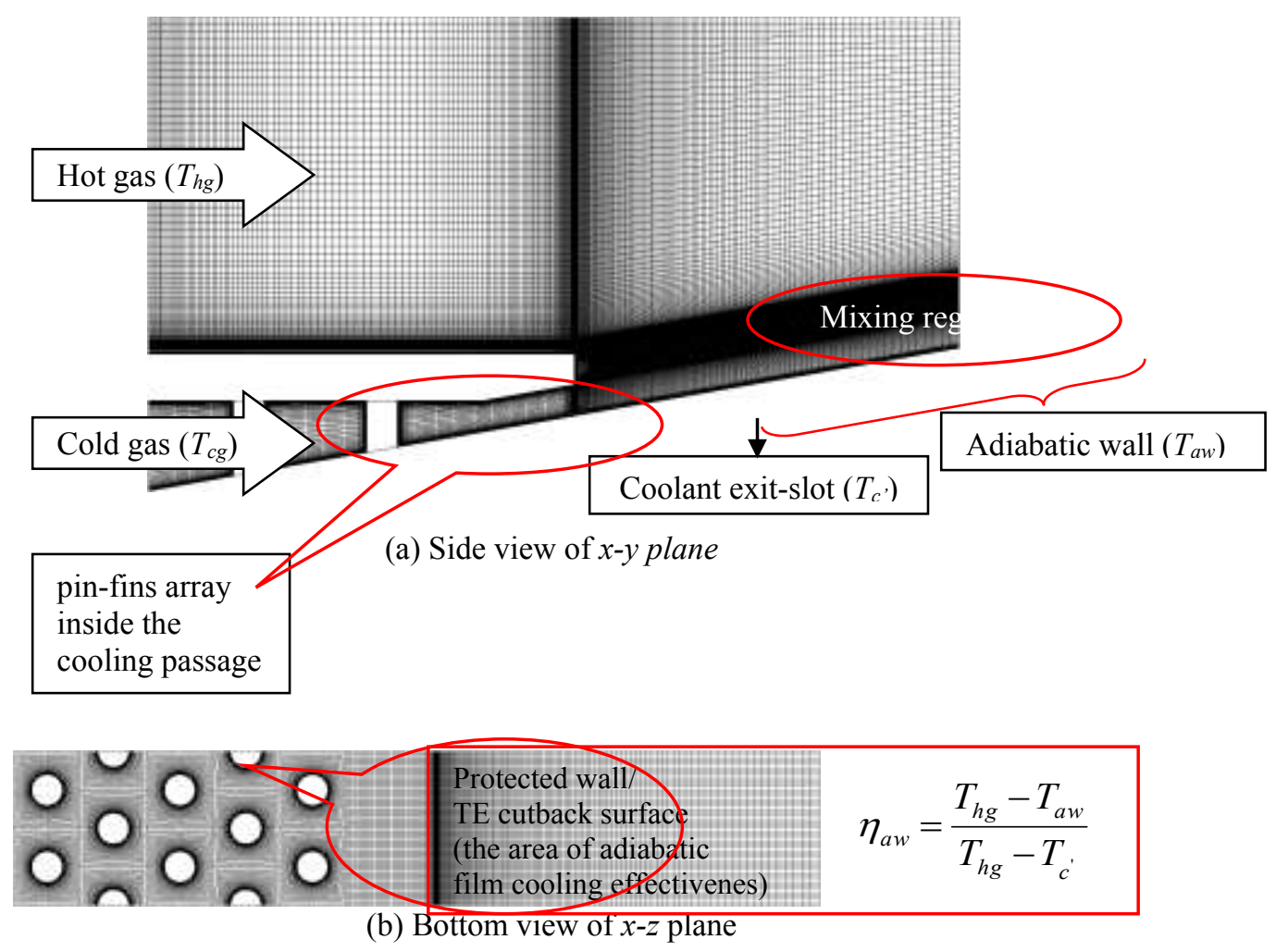

Fig. 1. Fine mesh for the baseline model.

\subsection{Algorithm}

A finite-volume method is utilised to solve the governing equations for incompressible flow. The equations are spatially computed using second-order accuracy on multi-block structured grids, whilst it is temporally calculated with a scheme of the second-order fully implicit. The Semi-Implicit Method for Pressure- 
Linked Equations Consistent (SIMPLEC) algorithm is chosen with second-order numerical scheme applied for all flow equations (i.e. pressure, momentum, energy) of the unsteady RANS and DES calculations. Not only the grid, but also the timestep size must be small enough in an attempt to guarantee a sufficiently fine temporal resolution of the unsteady flow effects. With respect to these requirements, small time-step sizes from $5 \times 10^{-5}$ to $1.25 \times 10^{-5}$ seconds are applied in these computations with considering the previous work carried out by Martini et al. [10].

\subsection{Adiabatic film-cooling effectiveness $\left(\bar{\eta}_{a w}\right)$, coefficient of discharge $\left(C_{\mathrm{D}}\right)$,} and blowing ratio $(\mathbf{M})$

Same formulas as seen in Martini et al. [10] are used to evaluate effectiveness, discharge coefficient and blowing ratios. These equations also have been presented in ref. [21] and [26].

\section{Results and Discussion}

\subsection{Validation against test data}

The grid refinement studies consider three successive meshes of the baseline domain from coarse to fine at a fixed-wall wall temperature $\left(T_{\mathrm{w}}\right)$ of $325 \mathrm{~K}$, as published by Effendy et al. [21]. The aim was to assess the adiabatic film-cooling effectiveness for three types of meshes, with considering DES simulation at lowand-high blowing ratios $(M=0.5$ and 1.1), in comparison with the experimental data reported by Martini et al. [10] and Horbach et al. [26]. It was realised to test the grid dependence for two different blowing ratios. In the paper highlighted that the DES modelling requires a fine resolution, which is fine enough to resolve the flow and heat transfer in the near end-wall region. Whilst results generated from the coarse mesh cause largely over-predict the film-cooling effectiveness near the downstream region. The use of the fine mesh produces results that are seen to be in a very good agreement with the test data along the protected/adiabatic wall surface from the slot-exit at $x / H=0$ to the downstream region of $x / H=12$, with the importance cooling effectiveness decay being captured successfully. The effect of meshes resolution is clear, indicated by low-blowing ratio case having largely deviation, as shown in ref. [21]. The deviation is reduced for the case with a higher blowing ratio.

\subsection{Discharge Coefficients $\left(C_{D}\right)$ predictions}

The discharge coefficient represents the discharge behaviour of the blade trailingedge cooling slots. This formula quantifies the global pressure loss within the cooling passage. It reveals the actual coolant mass flux to the ideal mass flux as the effect of an isentropic expansion from upstream region against free-stream at the ejection slot. Fig. 2 gives the predicted data of discharge coefficients for three different blowing ratios and various methods, in comparison with the experimental measurements. The predicted data are plotted versus the blowing ratios $(M)$. It has been found that simulation results using both steady/unsteady RANS and DES are in good agreement with the experimental data. This implies that the same properties of coolant inside the cooling passage could be predicted well for the use of both 
methods. The proximity data predicted for both manners justify the studies conducted by Martini et al. [10], which emphasise that there is no need for a costly DES analysis. This is due to the use of both steady/unsteady RANS and DES being in a close agreement in terms of predicting the discharge coefficients; however, the prediction will be more reliable if DES simulation is done for $C_{\mathrm{D}}$ prediction. As the mainstream remains constant for all various simulations during this study, the increase of blowing ratio provided by raising the coolant flow velocity at the inlet causes the increase of Reynolds number at the slot-exit. The change of blowing ratio is proportional to the change of the real coolant mass flow. The discharge coefficient is increased slightly by increasing the blowing ratios. This finding is consistent with the works of Martini et al. [5], which show that the coefficient of discharge is increased by raising the Reynolds number at the coolant flow. This invention confirms the right finding of adiabatic film-cooling effectiveness, which also has a sound suitability result, as discussed above. Hence, three cases studies of validation, using the baseline, are reasonable for development for further investigation.

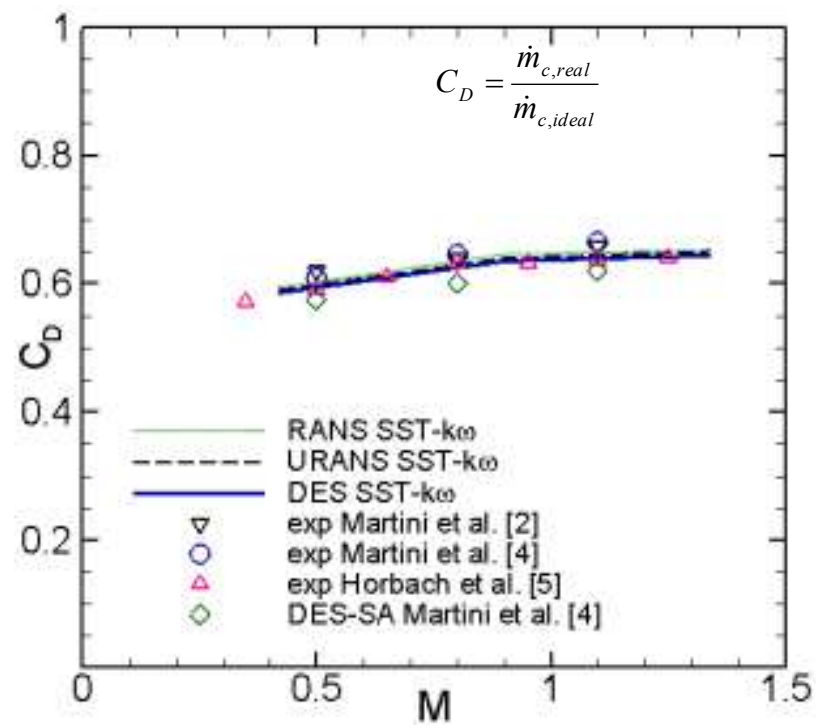

Fig. 2. Discharge coefficient.

\subsection{Adiabatic film-cooling effectiveness $\left(\bar{\eta}_{a w}\right)$ predictions}

Fig. 3 shows the quantitative prediction data of the adiabatic film-cooling effectiveness along the adiabatic/protected wall surface at various blowing ratios, in comparison with experimental measurements and previous numerical results using DES-SA [10]. It has been found that the SST $k-\omega$ turbulence is capable of being applied for prediction on eddy simulation. CFD data follows a strong decay of the adiabatic film-cooling effectiveness, much the same as with the experimental measurements. The decay is reduced by increasing the blowing ratio. Anomalous phenomenon occurred when the blade TE cutback cooling is simulated around medium blowing ratio $(M=0.8)$. There is an opposite order of film-cooling 
effectiveness trend. The adiabatic film-cooling effectiveness is decreased at the downstream region by increasing a blowing ratio. This finding is similar to the previous simulation carried out by Martini et al. [20] and in the experiment by Horbach et al. [26] Both previous data indicated that the adiabatic film-cooling effectiveness, at the highest blowing ratio, is less than at the medium blowing ratio-mainly at the middle region $4<x / H<8$. Horbach et al. [26]] noted that the adiabatic film-cooling effectiveness dropped by increasing the blowing ratios from $M=0.8$ to 1.25 . This decrease is due to the intensified vortex shedding from the ejection lip. It occurs within a certain operating range with slot ejection, leading to an intensified mixing process between coolant and mainstream.

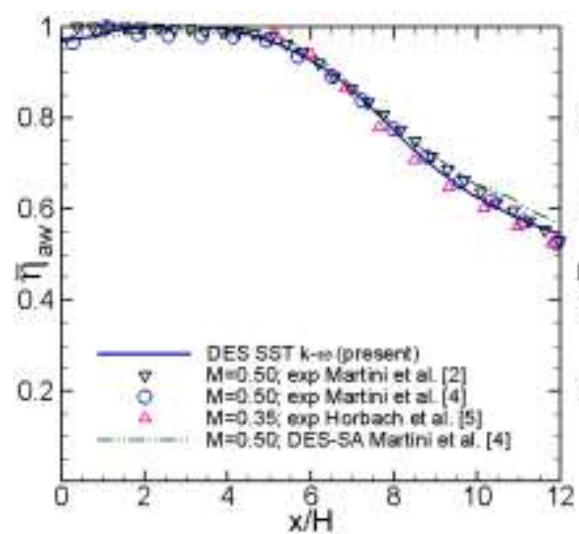

(a) Low $(M=0.5)$

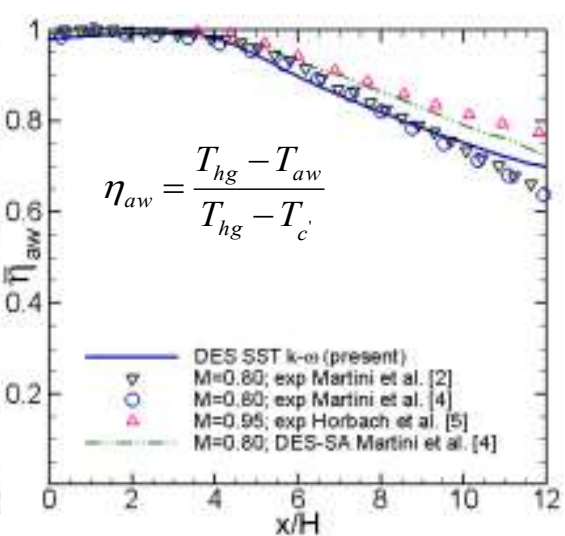

(b) Medium $(M=0.8)$

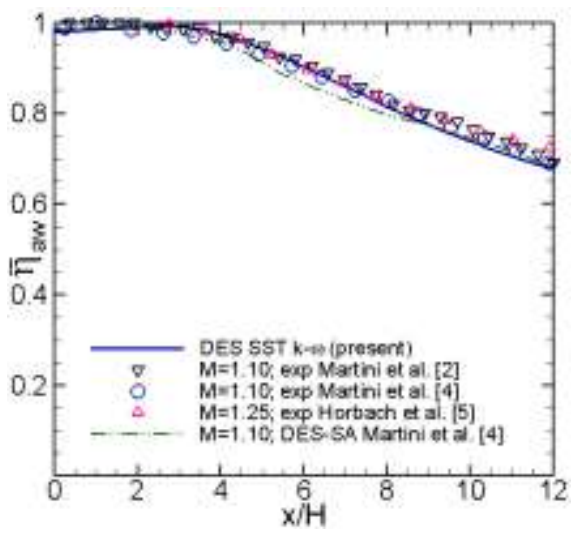

(c) High $(M=1.1)$

Fig. 3. Cooling-film effectiveness at various blowing ratios.

Fig. 4 presents a quantitative comparison of the averaged film-cooling effectiveness for both TE cutbacks. It has been found that each case study has a typical characteristic of film-cooling effectiveness, which is depending on their blowing ratios. A rapid decay of film-cooling effectiveness occurs for the simulation at low blowing ratios (e.g. $M=0.42$ ), whilst it tends to at a higher level for the simulation at high blowing ratios (e.g. $M=1.83$ ). This finding is consistent 
with the previous eddy-simulation carried out by Martini et al. [26]. It is instructive to briefly recall that the results of verification and validation using a TE cutback with single-pitch domain, which have been described in Fig. 4. The CFD prediction matches with the experimental data for three different blowing ratios tested (i.e. $M$ $=0.5,0.8$ and 1.1). The CFD prediction of the current case also agrees well compared to both the work of Martini et al. [10] and Horbach et al. [26].

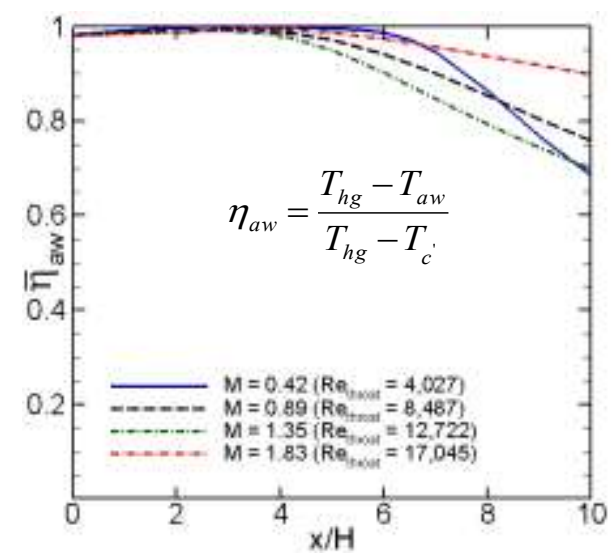

Fig. 4. Cooling-film effectiveness at various blowing ratios.

\subsection{Velocity profiles}

Fig. 5 shows the normalised $u$ velocity profiles of the stream wise velocity at different blowing ratios within selected downstream distance of $x / H=2,4,6,8,10$ and 12 . These are based on the averaged $u$ velocity at span wise direction (along the width of domain at a specific $z$-axis direction depending on the $x / H$ position). It has been found that the blowing ratio is a key role parameter to play the dynamic mixing process along the breakout region. It is evidenced by a stronger flow velocity when blowing ratio is increased from 0.42 to 1.83 . The left-curve and the right-curve discrepancy of the averaged $u$ velocity near the protected wall are noticeable as indicated by Fig. 5(a). The change of $u$ velocity profile is more pronounced around the slot exit region, for example at $x / H=2$. The distance of the left-curve and the right-curve reduces near the downstream region, for instance at $x / H=12$ (see Fig. 5(a)). 


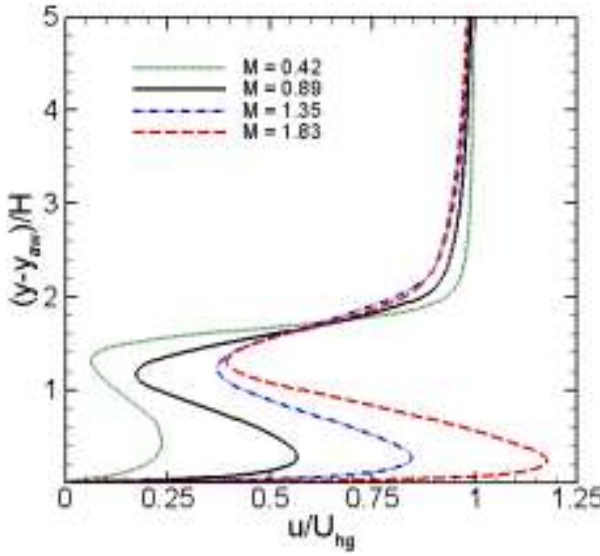

(a) $x / H=2$

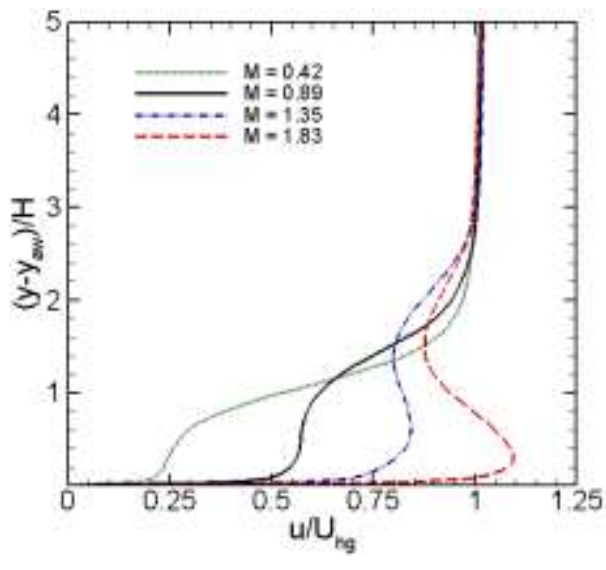

(c) $x / H=6$

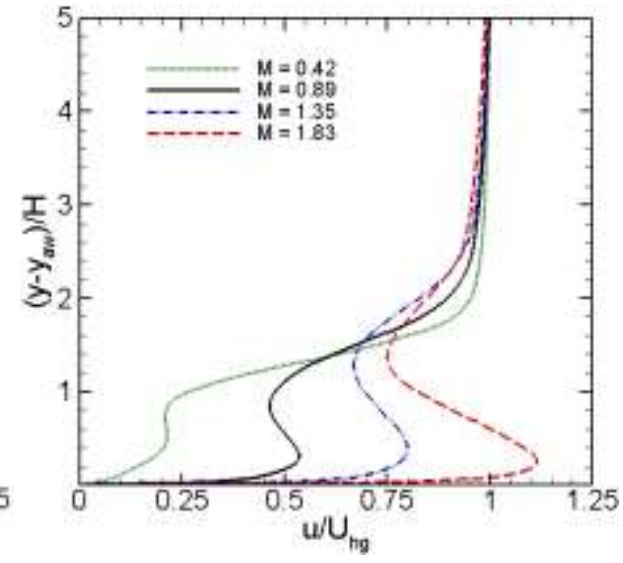

(b) $x / H=4$

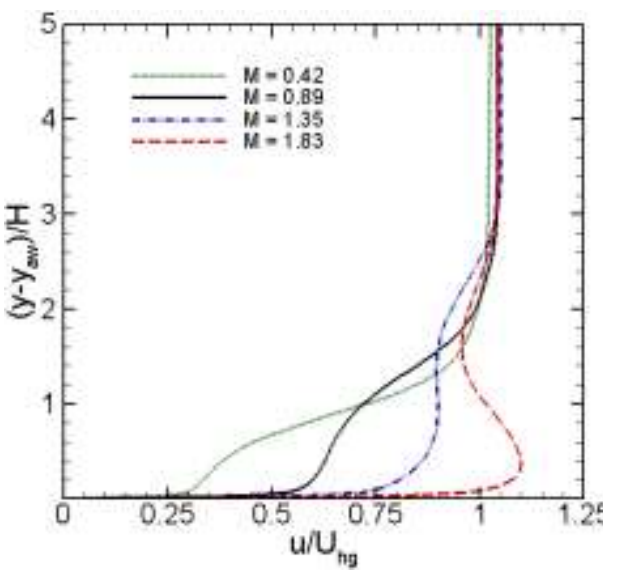

(d) $x / H=8$ 


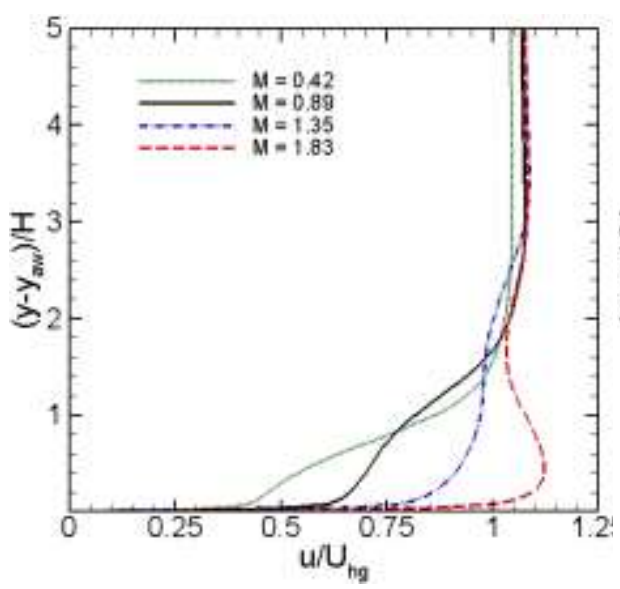

(e) $x / H=10$

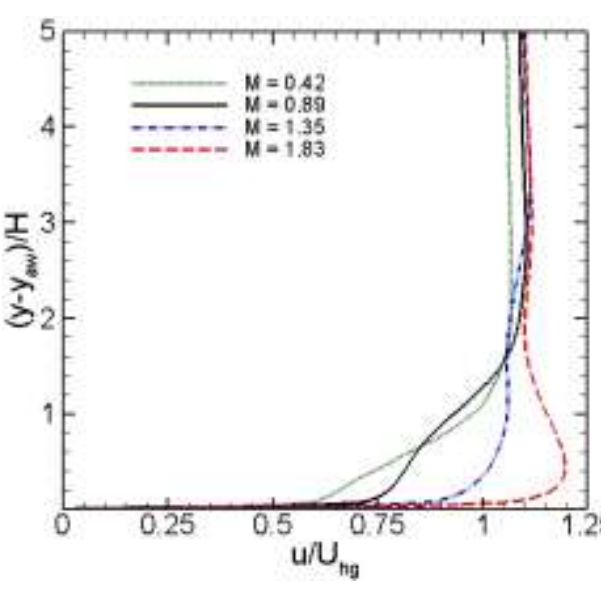

(f) $x / H=12$

Fig. 5. Normalised $u$ velocity profiles at different downstream locations.

\subsection{Strouhal number}

In terms of the Strouhal number, it has been recognised that a Strouhal number $\left(\mathrm{S}_{\mathrm{t}}\right)$ of vortex shedding is approximately 0.22 , which is corresponding to the shedding frequency of $2.21 \mathrm{kHz}$. It is suitable to previous computation study with $\mathrm{St}=0.24$. Sieverding et al. [25] report that vortex shedding of a turbulent boundary layer flow over a flat plate with a squared trailing edge is around $S_{\mathrm{t}}=0.21$. Boldman et al. [26] found that a Strouhal number of turbulent flow behind a blunt trailing edge is 0.2.

\section{Conclusion}

Overall, the predicted data of the adiabatic film-cooling effectiveness and the discharge coefficient are in good agreement for all various blowing ratios. It is proven by capturing well flow structures with the characteristic of shedding frequency and a Strouhal number approximately at $f=2.21 \mathrm{kHz}$ and $S_{\mathrm{t}}=0.22$, respectively. DES based on the SST $k-\omega$ turbulence model, is strongly recommended for prediction simulations compared to both steady and/or unsteady RANS. It is due to the fact that the flow structures of gas-mixing between the coolant and the mainstream flow could be captured more realistically.

\section{References}

[1] J. Choi, S. Mhetras, J. -C. Han, S. Lau et R. Rudolph, «Film Cooling and Heat Transfer on Two Cutback Trailing Edge Model with Internal Performance Blockages,» J. Heat Transfer, vol. 130, no.11, p. 012201, 2008.

[2] J. -C. Han, «Fundamental Gas Turbine Heat Transfer,» J. Thermal Science and Engineering Applications, vol. 5, no.12, pp. 021007-1, 2013.

[3] J. -C. Han et A. P. Rallabandi, «Turbine Blade Film Cooling Using PSP 
Technique,» Frontier in Heat and Mass Transfer, vol. 1, p. 1-21, 2010.

[4] S. D. Holloway, J. H. Leylek et F. A. Buck, «Pressure-Side Bleed Film Cooling: Part I - Steady Framework For Experimental And Computational Results,» chez ASME paper No GT-2002-30471, 2002.

[5] P. Martini, A. Schulz, C. F. Whitney et E. Lutum, «Experimental and Numerical Investigation of Trailing Edge Film Cooling Downstream of a Slot with Internal Rib Arrays,» chez Proc. Inst. Mech. Eng., Part A, 217, 2003.

[6] M. Effendy, Y. Yao et J. Yao, «Comparison Study of Turbine Blade with Trailing-Edge Cutback Coolant Ejection Designs,» chez 51st AIAA Aerospace Sciences Meeting, Texas, 2013-0548, 2013.

[7] Y. Egorov, F. R. Menter, R. Lechner et D. Cokljat, «The Scale-Adaptive Simulation Method for Unsteady Turbulent Flow Predictions. Part 2: Application to Complex Flows,» J. Flow, Turbulence and Combustion, vol. 85, no.11, p. 139-165, 2010.

[8] G. Medic et P. A. Durbin, «Unsteady Effects on Trailing Edge Cooling,» $J$. Heat Transfer, vol. 127, no.14, p. 388-392, 2005.

[9] J. Joo et P. Durbin, «Simulation of Turbine Blade Trailing Edge Cooling,» J. Fluids Engineering, vol. 131, no.12, pp. 021102-1 - 021102-14, 2009.

[10] P. Martini, A. Schulz, H. -J. Bauer et C. F. Whitney, «Detached Eddy Simulation of Film Cooling Performance on The Trailing Edge Cutback of Gas Turbine Airfoils,» J. Turbomachinery, vol. 128, no.12, p. 292-300, 2006.

[11] J. Krueckels, M. Gritsch et M. Schnieder, «Design Consideration and Validation of Trailing Edge Pressure Side Bleed Cooling,» chez ASME Paper No. GT-2009-59161, 2009.

[12] K. Viswanathan, M. Schur, P. R. Spalart et M. Strelets, «Flow and Noise Predictions for Single and Dual Stream Beveled Nozzles,» J. AIAA, vol. 46, no.13, p. 601-626, 2008.

[13] H. Schneider, D. von Terzi et H. -J. Baurer, «Large-Eddy Simulations of Trailing-edge Cutback Film Cooling at Low Blowing Ratio,» J. Heat and Fluid Flow, vol. 31, no.15, p. 767-775, 2010.

[14] H. Schneider, D. von Terzi et H. -J. Baurer, «Turbulent Heat Transfer and Large Coherent Structures in Trailing-Edge Cutback Film Cooling,» J. Flow, Turbulence and Combustion, vol. 88, no.11-2, p. 101-120, 2012.

[15] A. Viswanathan et D. K. Tafti, «Detached-Eddy Simulation of Flow and Heat Transfer in Fully Developed Rotating Internal Cooling Channel with Normal Ribs,» J. Heat and Fluid Flow, vol. 27, no.11, p. 351-370, 2006.

[16] A. Viswanathan, K. Klismith, J. Forsythe et R. Squires, «Detached-Eddy Simulation around a Forbody at High Angle of Attack,» chez AIAA Paper No. 2003-263, 2003.

[17] A. Viswanathan et D. K. Tafti, «Detached-Eddy Simulation of Turbulent Flow and Heat Transfer in a Two-Pass Internal Cooling Duct,» J. Heat and Fluid Flow, vol. 27, no.11, p. 1-20, 2006.

[18] S. D. Holloway, J. H. Leylek et F. A. Buck, «Pressure-Side Bleed Film Cooling: Part II - UnSteady Framework For Experimental and Computational 
Results,» chez ASME paper No GT-2002-30472, 2002.

[19] Y. Chen, C. G. Matalanis et J. K. Eaton, «High Resolution PIV Measurements around a Model Turbine Blade Trailing Edge Film Cooling Breakout,» $J$. Experiments in Fluids, vol. 44, no.12, p. 199-209, 2007.

[20] P. Martini, A. Schulz et H. -J. Bauer, «Film Cooling Effectiveness and Heat Transfer on The Trailing Edge Cutback of Gas Turbine Airfoils with Various Internal Cooling Designs,» J. Turbomachinery, vol. 128, no.11, p. 196-206, 2006.

[21] M. Effendy, Y. Yao, J. Yao et D. R. Marchant, «DES study of blade trailing edge cutback cooling performance with various lip-thicknesses,» J. Applied Thermal Engineering, vol. 99, pp. 434-445, 2016.

[22] T. Nishino, G. T. Roberts et X. Zhang, «Unsteady RANS and Detached-eddy Simulations of Flow around a Circular Cylinder in Ground Effect,» J. Fluids and Structures, vol. 24, no.11, p. 18-33, 2008.

[23] P. R. Spalart, «Young-Person's Guide to Detached-Eddy Simulations Grids,» Boeing Commercial Airplane Group, Seattle, WA USA, 2001.

[24] P. R. Spalart, S. Deck, M. L. Shur, K. D. Squires, M. K. Strelets et A. Travin, «A New Version of Detached Eddy Simulation, Resistant to Ambiguous Grid Densities,» J. Theoretical and Computational Fluid Dynamics, vol. 20, no.13, p. 181-195, 2006.

[25] F. R. Menter, M. Kuntz et R. Langtry, «Ten Years of Industrial Experience with the SST Turbulence Model,» Turbulence, Heat and Mass Transfer 4 , edited by K. Hanjalic, Y. Nagano, and M. Tummers, Begell House, Inc, p. 625632, 2003.

[26] T. Horbach, A. Schulz et H. -J. Bauer, «Trailing Edge Film Cooling of Gas Turbine Airfoils - External Cooling Performance of Various Internal Pin Fin Configurations,» J. Turbomachinery, vol. 133, no.14, pp. 041006-1 - 0410069, 2011.

[27] C. H. Sieverding et H. Heinemann, «The Influence of Boundary Layer State on Vortex Shedding from Flat Plates and Turbines Cascades,» J. Turbomachinery, vol. 112, no.12, p. 181-187, 1990.

[28] D. R. Boldman, P. F. Brinich et M. E. Goldstein, «Vortex Shedding from a Blunt Trailing Edge with Equal and Unequal External Mean Velocities,» J. Fluid Mechanics, vol. 75, no.14, p. 721-735, 1976. 